\title{
Suizid und Ehre in Schnitzlers Erzählung Spiel im Morgengrauen
}

\author{
Suicide and Honor in Schnitzler's Story "Night Games"
}

\author{
Matthias Schöning
}

\begin{abstract}
The following article focuses on suicide as a method to retrieve one's honour. Arthur Schnitzler's novella Spiel im Morgengrauen serves as the foundation for this analysis. Firstly, the concept of honour as a cultural phenomenon - as far as it is represented within the diegesis - will be discussed. Then, within the framework of Georg Simmel's theory of honour, we will analyze how the text proceeds to develop and criticize the culture of honour at the turn of the 20th century. The constellation of characters in Schnitzler's text functions as a starting point to discover the difference between character and text.
\end{abstract}

\section{Keywords}

Schnitzler, Arthur, "Spiel im Morgengrauen", constellation of characters, honour, (Georg Simmel's) theory of honour, suicide, 1900 - at the turn of the 20th century 
Im Zentrum dieses Beitrags steht eine und nur eine historisch und handlungslogisch spezifische Form des Suizids - nicht ,der Suizid“ im Allgemeinen. Thema ist der Suizid aus Gründen der Ehrenrettung oder kurz: der Ehren-Suizid. Was damit gemeint ist, demonstriert ein Beispiel, Schnitzlers Erzählung Spiel im Morgengrauen.

Am Anfang der Rekonstruktion jenes Geschehens, das mit dem Ehren-Suizid endet, steht der ursächliche Kontrollverlust eines Leutnants beim Spiel. Ein Spiel ohne Grenzen trifft auf eine Kultur mit umso starreren Regeln (1). Im zweiten Teil wird die Ausgangssituation nachgetragen. Der Morgen, der dem riskanten Spiel vorausgeht, unterstreicht noch einmal, dass die Ehre im Zentrum des Textes steht (2.). Damit stellt sich die Frage, was „Ehre“ ist. Bevor eine Antwort gegeben werden kann, müssen jedoch die alternativen Handlungsmöglichkeiten skizziert werden, die es innerhalb der dargestellten Welt und gemäß deren Maßstäben gegeben hätte (3.). Erst wenn man sieht, dass die Ehre nur den ihr anhängenden Figuren als alternativlos erscheint, begreift man die Logik der „Ehre“, die Georg Simmel pointiert herausgearbeitet hat (4.). Auf diesen theoretischen Teil folgen noch zwei Abschnitte. Im vorletzten Teil geht es um die Konsequenzen für die literarische Kommunikation. Wenn die Figuren nicht wissen, was sie tun, müssen die Leser den Text umso besser verstehen (5.). Im letzten Schritt wird der Blick auf die Figurenkonstellation erweitert und ein bis dahin noch fehlendes Handlungselement einbezogen, um zu einem Schlussresümee zu kommen (6).

\section{Ein Spiel ohne Limit in einer Kultur mit starren Grenzen}

Arthur Schnitzlers späte Erzählung Spiel im Morgengrauen bietet die Möglichkeit, anhand der dargestellten Welt einen allein aus Gründen des Ehrenschutzes motivierten Suizid zu untersuchen. Der Ausdruck „anhand der dargestellten Welt“ ist in einem vollgültigen Sinne zu verstehen. Die dargestellte Welt von Schnitzlers Erzählung ist unstrittig fiktiv, aber um dargestellte Welt handelt es sich auch insofern, als hier mittels eines fiktionalen Erzähltextes fallweise dargestellt wird, was es in der realen Welt tatsächlich gibt - oder vielmehr gab, wenn man den Veröffentlichungszeitpunkt berücksichtigt.

Der Text erscheint 1927 bei S. Fischer zu einem Zeitpunkt, als die Ehrproblematik bereits weitgehend historisch geworden ist. Der Text denotiert die Historizität des Falls im Übrigen dadurch, dass er die Hauptfigur, den Leutnant Kasda, Spielschulden in einer Währung machen lässt, die nur bis 1900 im Umlauf war. Breits 1892 war der Gulden durch die Krone als österreichisch-ungarische Währung ersetzt worden. Um 1900, dem Jahr, in dem Schnitzlers Lieutenant Gustl, seine Erste, bekanntere und wichtigere „Leutnantsnovelle“ (Arthur Schnitzler) erschien, hat die Ehrpraxis noch den Alltag der Habsburger Doppelmonarchie mitgeprägt. Nach dem ersten Weltkrieg, dem Ende des Reiches und der Konstitution unabhängiger demokratischer Nationalstaaten ist es mit der kulturprägenden Funktion des Militärs und infolgedessen auch mit dem Ehrenkult vorbei. Schnitzler arbeitet seine zumeist noch in die ältere Zeit zurückreichenden Vorstudien aber gleichwohl noch aus - Erzählungen wie Spiel im Morgengrauen oder der noch spätere, erst postum 1932 veröffentlichte Text Der Sekundant. 
Doch nicht die Publikationspraxis alternder Autoren soll hier Thema sein, sondern Suizid und Ehre in Schnitzlers Erzählung: Es sind ausschließlich die Zwänge der Ehr-Praxis, die den Offizier Leutnant Willi Kasda dazu bewegen, seinem Leben von eigener Hand ein Ende zu setzen. Er benutzt dazu einen „Revolver“ (324). ${ }^{1}$ Der Anlass sind Spielschulden, die dank Sprichwort auch heute noch als Ehrenschulden geläufig sind. Die Spielschulden sind zudem sehr hoch. Der Betrag beläuft sich auf 11000 Gulden, während das Gehalt eines Leutnants, der in der Kaserne wohnt und einen Burschen hat, seinerzeit 70 Gulden betrug. ${ }^{2}$

Leutnant Kasda, die Hauptfigur, die sich am Ende erschießen wird, handelt unverantwortlich. Das ist unstrittig. Bereits kurz nachdem er den Spielort, ein Café im vor den Toren Wiens gelegenen Baden, betreten hat, heißt es angesichts der getätigten Einsätze: „Das ist heute wirklich nichts für mich, dachte Willi und schob seinen Sessel noch weiter zurück“ (244). Und auch zu Beginn des entscheidenden Spiels ist klar, dass der Leutnant, aber auch andere Beteiligte weit über ihre Verhältnisse leben bzw. spielen:

Der Konsul legte eine Bank auf, wie sie in diesem Kreise noch nicht erlebt worden war, eine Bank von dreitausend Gulden. Außer der Spielergesellschaft und einem Kellner befand sich kein Mensch mehr im Café. Durch die offenstehende Tür drangen von draußen her morgendliche Vogelstimmen. Der Konsul verlor, aber er hielt sich vorläufig mit seiner Bank. Elrief hatte sich vollkommen erholt, und auf einen mahnenden Blick des Fräulein Rihoschek zog er sich vom Spiel zurück. Die anderen, alle in mäßigem Gewinn, setzten bescheiden und vorsichtig weiter. Noch war die Bank zur Hälfte unberührt (257).

Das Spiel entgleist, weil Leutnant Kasda seine Spontaneität nicht mehr im Griff hat. Er hätte bereits mehrfach mit einem hohen Gewinn nach Hause fahren können. Nachdem dieser aber zusehends wieder zusammengeschmolzen ist, verliert er vollends die Kontrolle. Das bleibt am Spieltisch auch nicht unbemerkt. Der Regimentsarzt interveniert sogar explizit. Aber die Zusammensetzung der Spielrunde ist gegenseitiger Rücksichtnahme nicht förderlich. Den stolzen Vertretern des Militärs steht oder vielmehr sitzt ein „Konsul Schnabel“ gegenüber, von dem man „nicht viel mehr“ weiß, „als daß er eben Konsul war, Konsul eines kleinen Freistaats in Südamerika und ,Großkaufmann““ (245). Geld trifft auf Ehre könnte man zugespitzt sagen. Ob der Konsul nämlich satisfaktionsfähig ist, wird explizit in der Schwebe gelassen. Er ist finanziell zu potent und seine Aura zu aggressiv, als dass man sich trauen würde, ihm tatsächlich entgegenzutreten. So deutet Schnitzler mittels Figurenkonstellation an, dass nicht nur das Leben des verschuldeten Leutnants auf dem Spiel steht, sondern sich die Kultur der Ehre ihrem Ende entgegen neigt.

Es zeigt sich, was der Text als sein Bezugsproblem exponiert. Er konfrontiert die militärische Kultur der Ehre mit ihrem Kult um die Satisfaktionsfähigkeit mit solchen

1 Zitiert wird nach der Ausgabe Arthur Schnitzler: Die großen Erzählungen, hg. v. SCHEFFEL, Michael, Stuttgart 2006, S. 225-326.

2 Vgl. den Kommentar von Ursula Renner in ihrer Ausgabe Arthur Schnitzler: Lieutenant Gustl. Text und Kommentar, Frankfurt am Main 2007, S. 121. 
gesellschaftlichen Kräften, die sich im Medium des Geldes artikulieren. Auf hoch symbolische Weise lässt der Text einen Vertreter der Ehrkultur an der Geldkultur scheitern. Für sein Scheitern ist der besagte Leutnant selbst verantwortlich. Dass er glaubt, danach gar nicht mehr weiterleben zu können, sagt jedoch weniger etwas über ihn als über die Kultur aus, die er verkörpert. Eine Kultur, die einem zwar hoch, aber auch sehr kurzfristig Verschuldeten keinen anderen Ausweg lässt, als dem eignen Leben binnen Stunden ein Ende zu setzen, ist selbst am Ende - zumal wenn um den Betroffenen herum Geld in Hülle und Fülle vorhanden ist.

So endet das Geschehen denn auch: Nachdem es Kasda nicht gelungen ist, das Geld bis zu der vom Gläubiger gesetzten Frist aufzutreiben, hat er nur zwei Möglichkeiten. Er steht vor der Alternative, seinem Leben als Offizier und d.h. als Angehöriger des Standes, der die Kultur der Ehre pflegt, ein Ende zu setzen oder den Dienst zu quittieren und entehrt weiterzuleben. Leben und Ehre treten durch die Spielschulden in ein kontradiktorisches Verhältnis. Sie schließen einander aus. Entweder Ehre - und das heißt Tod bis zur Frist - oder Leben - und das heißt Austritt aus der Korporation, der er seine Ehre verdankt, nämlich dem Offizierskorps. Adelig ist Kasda nicht. Er genießt Ehre und unterliegt ihren Regeln nur wegen Zugehörigkeit zum Offizierskorps.

Beiläufig sei schon einmal festgehalten, dass es sich bei dieser Ehre im engeren Sinne um soziale Regeln handelt, die es nur in Verbindung mit einer bestimmten Form von Gruppenbildung gibt. Ehre in diesem engeren Sinne ist an Korporationen gekoppelt, die mittels Ehre eine scharfe Grenze zwischen den Gliedern des Gemeinschaftskörpers und ihrer Umwelt ziehen.

\section{Initiierung der Handlung und Exposition des Themas: Willi, Otto und die Kontaktregeln der Ehr-Kultur}

Nun könnte man sich fragen, warum es überhaupt so wichtig ist, hier die Ehre als das gefährdete Gut ins Zentrum zu stellen. Will der Leutnant Willi Kasda nicht einfach seinen Beruf behalten? Einen anderen hat er ja nicht. - Drei charakteristische Situationen, die es zugleich ermöglichen, die fehlenden Teile der Handlung kurz zu rekapitulieren, markieren deutlich, dass es eine der zwei zentralen Textintentionen ist, die Schärfe der Trennlinie zwischen Menschen mit und ohne Satisfaktionsfähigkeit, mit und ohne Ehre zu problematisieren.

Der dominant heterodiegetisch erzählte Text beginnt szenisch. Der Leser muss die tiefgreifende Problematik der Situation aus Dialogen und Gedankenrede rekonstruieren. Zu Schnitzlers Zeiten hat das sicher reibungslos funktioniert. Wir Heutigen müssen uns die partiell fremd gewordenen Denk- und Handlungsvoraussetzungen der Zeit vor dem Ersten Weltkrieg jedoch erst bewusst machen: Die Hauptfigur, der zunächst nur „Wilhelm“ (227) genannte Leutnant Kasda, wird an einem frühen Sonntagmorgen von seinem Burschen geweckt, weil ein problematischer Besucher vorstellig wird. Es handelt sich um den ehemaligen Regimentskameraden Herrn von Bogner. Dieser ist zwar adelig, wurde aber „unter peinlichen Umständen“ (322) aus dem österreichischen Of- 
fizierskorps „verabschiedet“ (322), d.h. also unehrenhaft entlassen, weil er „Schulden“ (228) nicht bezahlen konnte. Nun ist er neuerlich in finanziellen Nöten. Er arbeitet „in einem Büro für elektrische Installation [...] als Kassierer“ und hat sich „etliche Male aus der Kasse was ausleihen müssen“ (231) wie er selbst sagt. Während der Grund für seine Veruntreuungen zunächst Arztkosten waren, also gleichsam ,ehrbare Gründe, bleibt unklar, warum es „diesmal [...] ein bissl mehr geworden [ist] als sonst, leider“. (231) Er bittet seinen ehemaligen Regimentskameraden, den er nun, nachdem die Karten auf dem Tisch liegen, nicht nur mit seinem Vornamen, sondern der Koseform „Willi“ anspricht (231), ihm das benötigte Geld zu leihen, da für den kommenden Montag „eine Revision" (231) angesetzt ist.

Otto bewegt sich nicht nur monetär, sondern auch sozial am Rande des Offenbarungseides. Er muss ein Geständnis ablegen, das höchst unangenehm ist, weil es einen tiefen Einblick in die Person mit sich bringt, während die kulturellen Verkehrsformen eigentlich das Gegenteil verlangen. Wechselseitige Interaktion, zumal außerhalb des engsten Familienkreises, mag zwar sekundär von Einschätzungen der Person mitgeprägt werden, primär orientiert sie sich am Sozialprofil der Rolle, die jemand verkörpert. So gesehen ist Otto sozial nackt. Dass er sein Anliegen überhaupt vorbringt und sich gleichsam erdreistet, an einem Sonntagmorgen in die Kammer des Leutnants einzudringen, impliziert, dass ein seltenes Vertrauensverhältnis besteht oder soweit einmal bestand, dass es Aussicht auf Erneuerung gibt. Otto und Willi müssen einmal eng befreundet und nur durch Ottos unehrenhaftes Ausscheiden getrennt worden sein. Zur Freundschaft gehört es sogar dazu, den anderen nicht zu behelligen, wenn man seine Ehre verliert, denn das brächte diesen nur in Loyalitätskonflikte. Solche Rücksicht wird explizit, wenn Otto, trotz beiderseitigem Bedauern darüber, dass ihre Freundschaft seit Ottos Ehrverlust keine Zukunft mehr hat, beteuert, dass er sicher dabei geblieben wäre, „nichts [...] von [sich] hören und sehen [zu] lassen“ (230), wenn es nicht seine absolut letzte Wahl wäre.

Andernfalls bleibe ihm sonst nichts anderes übrig als seinem Leben ein Ende zu setzen - „was vielleicht schon vor zwei Jahren das Gescheiteste gewesen wäre“ (230), wie er bitter anfügt. Diese Stelle erlaubt es, eine wichtige Differenzierung vorzunehmen: Auch jemand, der seine Satisfaktionsfähigkeit verloren hat und insofern außerhalb der Ehrkultur steht, kann an Selbstmord denken. Der Suizid ist kein Privileg ständischer Ehrkultur - natürlich nicht. Der Unterschied ist jedoch, dass es hier um einen Suizid aus existentieller Verzweiflung ginge, während der Ehren-Suizid aus sozialer Verzweiflung heraus erfolgt. Für Otto geht es um die grundlegendsten Subsistenzbedingungen - die historische Verhaltenserwartungen und Geschlechternormen vorausgesetzt. Wenn er die benötigten 960 Gulden nicht auftreiben kann, wird er entlassen, verklagt, ggf. inhaftiert und findet sicher keine Arbeit mehr, mit der er seine Familie ernähren kann. Zwei Jahre zuvor, als er in der Situation war, in der Kasda am Ende des Geschehens sein wird, stand er vor der Wahl zwischen zwei sozialen Rollen. Wer sich ein Leben als entehrter Ex-Offizier vorstellen kann, muss sich nicht umbringen. Das führen neben diesen zwei Protagonisten zahlreiche weitere Figuren anderer Texte von Schnitzler vor.

Anders als Willi am Ende des dargestellten Geschehens hat Otto also tatsächlich keine andere Wahl und nur diese biographische Position nahe an einem Abgrund, 
hinter dem es keine Lebensalternative mehr gibt, rechtfertigt ihn, bei Willi vorstellig zu werden. Beiden Gesprächspartnern ist demnach bewusst, dass Otto von Willi sehr viel verlangt. Trotzdem wird er nicht abgewiesen. Willi verfügt zwar nicht länger über die Geldquelle, die Otto unterstellt, nämlich seinen Onkel Robert, aber er sagt zu, das Geld für ihn erspielen zu wollen. Wie das ausgeht, wissen wir bereits.

Zur Beantwortung der Frage, warum es wichtig ist, hier die Ehre als das gefährdete Gut ins Zentrum zu stellen, war der Vergleich zwischen einer etwaigen Suizidmotivation Ottos im Unterschied von der Willis durchaus hilfreich. Willi hat am Ende der dargestellten Handlung immer noch mehr Optionen als Otto jetzt. Er wird dann nicht existentiell verzweifelt sein, sondern verzweifelt über den drohenden Verlust des Offiziersstatus und der damit verknüpften Ehre.

Auch ein Rückblick auf die Details der ersten Sequenz der Erzählung belegt, dass die Ehre im Fokus steht. Denn bevor es zu der unverantwortlichen und dann auch entsprechend folgenreichen Zusage kommt, das Geld erspielen zu wollen, muss der aktive Leutnant Wilhelm Kasda den unehrenhaft entlassenen Ex-Oberleutnant Otto von Bogner erst einmal empfangen. Erst ex post, nachdem deutlich geworden ist, wie vertrauensvoll die Beziehung der beiden Personen einmal gewesen sein muss, wird deutlich, wie trennend die verlorene Ehre ist. Es hätte nur wenig dazwischen kommen müssen und die verlorene Satisfaktionsfähigkeit Ottos hätte eine Begegnung verhindert. Dann hätte Otto unverrichteter Dinge, ohne seine Bitte überhaupt aussprechen zu können, wieder gehen müssen - mit der weiteren Konsequenz, dass am Ende er sich per Verzweiflungs-Suizid selbst richtet und nicht Willi per Ehren-Suizid - wenn eine solche kontrafaktische Überlegung gegenüber einem fiktiven Szenario erlaubt sein mag.

Ein längeres Zitat belegt, wie sehr die Ehr-Logik die ganze Handlung steuert:

Der Leutnant, sich im Bette halb aufrichtend, vermochte nun den Namen auf der Karte zu lesen, ließ sie auf die Bettdecke sinken, betrachtete sie nochmals, kraute sein blondes, kurz geschnittenes, morgendlich zerrauftes Haar und überlegte rasch: »Abweisen? - Unmöglich! Auch eigentlich kein Grund. Wenn man wen empfängt, das heißt ja noch nicht, daß man mit ihm verkehrt. Übrigens hat er ja nur wegen Schulden quittieren müssen. Andere haben halt mehr Glück. Aber was will er von mir? - Er wandte sich wieder an den Burschen: »Wie schaut er denn aus, der Herr Ober -, der Herr von Bogner?"

Der Bursche erwiderte mit breitem, etwas traurigem Lächeln: »Melde gehorsamst, Herr Leutnant, Uniform ist dem Herrn Oberleutnant besser zu G'sicht gestanden."

Wilhelm schwieg eine Weile, dann setzte er sich im Bett zurecht: »Also, ich laß bitten. [...]《(S. 227-228).

Der heikle Punkt ist mehr als nur angedeutet: „Wenn man wen empfängt, das heißt ja noch nicht, daß man mit ihm verkehrt." Hinter dieser subtilen Unterscheidung verbirgt sich die Differenz zwischen zwei Ständen, die kaum größer sein könnte. Unter den Denkvoraussetzungen seines Standes und dessen Ehrenkodexes vollkommen korrekt unterscheidet der Leutnant Kasda zwischen asymmetrischen und symmetrischen Sozialkontakten. Jemanden empfangen, jemanden vorlassen, ist ein asymmetrischer Vorgang 
bei dem die höher gestellte Person der anderen eine Gnade erweist, die durch keinerlei Rechtsmittel erwirkt werden könnte. Es hängt ganz von der höhergestellten Person ab, ob sie sich dazu herablässt, die niedriger gestellte Person zu empfangen. Mit jemandem zu verkehren, bezeichnet dagegen symmetrische Sozialkontakte zwischen Personen, die eine gleiche soziale Wertigkeit besitzen oder sich wenigstens situativ zuschreiben. Hier spielt beides ineinander. Als erstes sorgt der Leutnant sich, dabei beobachtet zu werden, nicht standesgemäßen Besuch zu empfangen. Im zweiten Schritt beruhigt er sich darüber mit der zitierten Subtilität, um dann drittens schließlich in ein eindeutig symmetrisches Verhältnis zu Otto treten. Dass er sich die Anrede mit der Koseform „Willi“ gefallen lässt, zeigt an, dass er die öffentlich geforderte Distanz im privaten Binnenraum nicht im mindesten aufrechtzuerhalten in der Lage ist. Er sagt nicht nur zu, einen Hilfsversuch zu unternehmen und erweist sich dabei - vom Ende her betrachtet - als redlicher Freund. Zudem macht Willi sich Otto dadurch gleich, dass er das Geld im Spiel zu gewinnen hofft, also auf jenem Wege, mit dem Ottos Abstieg begann. Sorgen muss man sich also nicht nur wegen dieser Parallelität machen, sondern auch deshalb, weil sich bereits hier zeigt, dass Leutnant Kasda keineswegs so prinzipienfest ist, wie es sein Stand eigentlich von ihm fordert. Diese Eigenschaft, die für das Spiel um Geld eine schlechte Voraussetzung ist, teilt er mit dem anderen Leutnant aus Schnitzlers Feder, dem Leutnant Gustl.

Als am Ende der Erzählung Otto von Bogner und der Regimentsarzt vor der verschlossenen Kammertür aufeinandertreffen, hinter der sie bald darauf den toten Leutnant finden werden, werden die von der Ehr-Logik gesteuerten Rang- und Kontaktregeln noch einmal in Erinnerung gerufen:

Während Joseph [der Bursche des Leutnants] nach unmerklichem Zögern an die Tür pochte, warf Tugut [der Regimentsarzt] einen mißtrauischen Blick auf den Zivilisten, der im Vorraum stand- Bogner stellte sich vor. Der Name des unter peinlichem Umständen verabschiedeten Offiziers war dem Regimentsarzt nicht unbekannt, doch tat er nichts dergleichen und nannte gleichfalls seinen Namen. Von Händedrücken wurde abgesehen (322).

Hier wird das Trennende des Ehrenkodexes abermals deutlich. Nicht die soziale Gruppe und ihre Regeln der Inklusion werden thematisch, sondern der Exklusivcharakter und bald danach auch die tödliche Gewalt, die dem Grenzregime der Ehre wesentlich ist. Nachdem die Tür mit Hilfe eines Schlossers geöffnet worden ist, bietet sich folgendes Bild:

Der Leutnant Willi Kasda, im Mantel mit hochgestelltem Kragen, lehnte in der dem Fenster zugewandten Ecke des schwarzen Lederdiwans, die Lider halb geschlossen, den Kopf auf die Brust gesunken, schlaff hing der rechte Arm über die Lehne, der Revolver lag auf dem Fußboden, von der Schläfe über die Wange sickerte ein schmaler Streifen dunkelroten Bluts, der sich zwischen Hals und Kragen verlor. So gefaßt sie alle gewesen waren, es erschütterte sie sehr (S. 324). 


\section{Implizite und explizite Handlungsalternativen? Von der Figur zum Text}

Um diesen Tod richtig zu verstehen, muss man sich die bereits skizzierten Alternativen und den entscheidenden Handlungsgrund vor Augen führen. Der Tod des Leutnant Kasda ist weder „die einzige Möglichkeit, dem gesellschaftlichen und moralischen Ruin zu entgehen“, noch kann man von einem „unaufhaltsamen Abstieg des Leutnants“ sprechen, der in dessen Selbstmord anschaulich finalisiert wird. Beides kann man im jüngst erschienenen Schnitzler-Handbuch ${ }^{3}$ lesen, ist aber falsch. Schnitzlers Text stellt die Alternativen zum Selbstmord mit größtmöglicher Deutlichkeit vor Augen. Man kann darüber streiten, ob die für Kasda bestehenden Alternativen als „explizit“ zu bezeichnen sind. An den Ansprüchen eines assertorischen Textes gemessen, sind sie sicher implizit, weil die Alternative nicht als Alternative bezeichnet wird. Gleichwohl dürfte unstrittig sein, dass Otto von Bogner als eine Art wandelnde Alternative fungiert. Mit den typischerweise an fiktionale Erzähltexte angelegten Maßstäben gemessen, kann man durchaus sagen, dass die Alternative explizit wird. Bogner personifiziert die Möglichkeit, den Dienst unehrenhaft zu quittieren und ein anderes Leben zu beginnen, wie wir bereits erörtert haben. Die wirtschaftlich erfolgreiche Variante dazu ist der Konsul, der ihm die vernichtende Frist setzt. Zu nennen ist aber auch die wichtige Frauenfigur Leopoldine Lebus-Wilram, über die noch zu sprechen ist. Sie alle zusammen differenzieren das Spektrum alternativer Lebensmöglichkeiten außerhalb der Ehrkultur weiter aus.

Dass Willis Tod alles andere als alternativlos ist, wird auch dadurch unterstrichen, dass das benötigte Geld noch vor der Frist eintrifft. Der Explizitheitsgrad der Alternativen wird abschließend noch einmal gesteigert. Nur weil Willi - und mit ihm dem Leser - bewusst vorenthalten wird, dass Rettung in Auftrag gegeben wurde, und er nicht die Kaltblütigkeit besitzt, bis zum allerletzten Moment zu warten, ist er bereits tot, als sein Onkel Robert als Geldbote Leopoldines eintrifft. Zuvor hilft Willi seinem alten Freund Otto mit einem letzten Tausender aus der Klemme. Er schickt seinen Burschen zu diesem Zweck weg und nutzt den Umstand, allein zu sein, um sich das Leben zu nehmen.

Nun stellt sich die Frage, was dieser Ehren-Suizid bedeutet. Man kann nicht sagen, dass es keinen Ausweg gebe. Das geht leicht über die Lippen, ist aber falsch, wie bereits festgehalten wurde. Mit der Kultur der Ehre geht ein strenges Reglement einher, doch dieses schließt die soziale Ordnung nicht vollständig ein. Im Gegenteil: Die Ehr-Kultur ist in weit fortgeschrittenen Gesellschaften wie dem deutschen und österreichich-ungarischen Kaiserreich eine Eliten-Kultur und als selektive Oberschichtenkultur prinzipiell eine Teilkultur, mittels der sich eine exklusive Gruppe von der Mehrheitsgesellschaft symbolisch abtrennt. ${ }^{4}$

3 CAGNEAU, Irène: „Spiel im Morgengrauen (1926/27)“, in: Christoph Jürgensen/Wolfgang Lukas/Michael Scheffel (Hgg.): Schnitzler-Handbuch. Leben - Werk - Wirkung, Stuttgart/Weimar 2014, S. 232-236, hier S. 233.

4 Vgl. - allerdings für Deutschland resp. Preußen - ELIAS, Norbert: Studien über die Deutschen. Machtkämpfe und Habitusentwicklung im 19. und 20. Jahrhundert, hg. v. SCHRÖTER, Michael: Frankfurt am Main 1990, S. $61-158$. 
Deshalb gibt es unter den Bedingungen der Ehre immer einen Ausweg, doch wird dieser nicht beschritten. Das ist der entscheidende Punkt, der einerseits für diesen Text wichtig ist und andererseits eine Verallgemeinerung ermöglicht. Wo literarische Texte Selbstmorde darstellen, die Ehrkonflikten assoziiert sind, sind drei Faktoren im Spiel. Die Hauptfigur wird in eine vordergründig aussichtslos wirkende Problemsituation geführt. Im Hintergrund zeigen Nebenfiguren und andere Träger semantischer Informationen Alternativmöglichkeiten auf. Die alternativen Handlungsoptionen werden jedoch nicht ergriffen. Stattdessen stürzt sich Hebbels Klara, die Hauptfigur seines Drama $M a-$ ria Magdalene, in den Brunnen, Fontanes Innstetten tötet Crampas im Duell und zerstört dadurch sein Lebensglück und das Leben seiner Frau, Effi Briest, und unser Leutnant jagt sich standesgemäß eine Kugel durch den Kopf.

Für diese drei Beispiele gilt: Es gäbe die Möglichkeit den Ehren-Kodex hinter sich $\mathrm{zu}$ lassen und davon unbeschwert weiter und oft besser weiterzuleben. Doch warum werden diese Möglichkeiten nicht ergriffen? Was bedeutet es, dass eine ganze Zahl an Texten, die sich mit Ehrkonflikten befassen, alternativlos wirkende Duelle und Suizide vor einem Horizont nicht ergriffener Möglichkeiten darstellen? Warum wird nicht der explizite Bruch mit der Ehr-Kultur in Szene gesetzt, sondern die finale und das heißt zugleich letale Selbstinklusion?

Zunächst eine methodologische Feststellung: Man versteht die Texte falsch, wenn man das dargestellte Geschehen von der Figur her perspektiviert. Das Textwissen von der Antiquiertheit der Ehrkultur ist vom eingeschränkten Figurenwissen strikt zu unterscheiden. Mit der paradoxen Ausnahme von „Leutnant Gustl“ wissen die Figuren nicht, was sie tun. Sie sind nicht in der Lage, über den Tellerrand der Ehr-Kultur zu schauen, die sie in einem falschen Selbstverständnis geradezu gefangen hält.

Auch die literarischen Verfahren Schnitzlers widersprechen dem lediglich auf den ersten Blick. Die Funktion der bei Schnitzler stark ausgeprägten Mitsicht bzw. Internen Fokalisierung und des mehr oder weniger dramatischen Modus ist nicht, Empathie für die Figuren zu erzeugen. Sie dient vielmehr dazu, die Begrenztheit des Horizonts nicht lediglich zu denotieren, sondern zu ,exemplifizieren' ${ }^{5}$ Wenn die Figuren vorführen oder beteuern, dass es keine Alternative gebe, muss man mit Texten rechnen, die das Gegenteil zeigen. Texte sind ihren Figuren gegenüber oft illoyal. Die Gründe dafür gilt es zu untersuchen, um die Textbedeutung zu rekonstruieren.

\section{Exkurs zu Georg Simmels soziologischer Rekonstruktion der Ehre-Logik}

Dass die Einschätzung der Situation durch die Figuren nicht vollständig richtig ist und vom Text als einseitig, beschränkt oder einfach falsch angezeigt wird, ist zunächst einmal schlicht und einfach sachadäquat. Folgt man Georg Simmel, dann ist es geradezu das Wesen der Ehre, dem Einzelnen „seine soziale Pflicht zu seinem individuellen Heile“ zu

5 GOODMANN, Nelson: Sprachen der Kunst. Entwurf einer Symboltheorie, übers. v. Bernd Philippi, Frankfurt am Main 1998, S. 59 f. 
machen. ${ }^{6}$ Das heißt, dass dem Einzelnen ein sozialer Zweck als individuelles Anliegen vorgegaukelt wird. Man kann sich die alles entscheidende Perspektivierungsrichtung, in der Simmel zu seiner höchst instruktiven Charakteristik der Ehre kommt, gar nicht deutlich genug vor Augen stellen. Simmel behandelt die Ehre im Kapitel 8 seiner Soziologie unter der Überschrift „Die Selbsterhaltung der sozialen Gruppe“. Er rekonstruiert gleichsam eine kollektive Intentionalität. Er versucht, sich in das Quasi-Bewusstsein sozialer Gruppen einzufühlen und von dort her deren Strategie, sich als Kollektiv zu erhalten, zu denken. Er perspektiviert ausdrücklich nicht von den Mitgliedern der Gruppe her, die ein gemeinsames Interesse zusammengeführt hat, sondern von einer gegebenen sozialen Institution, die nur ein Interesse hat, nämlich sich zu erhalten - egal, was das für die Angehörigen bedeutet.

In Simmels historisch und kulturell angemessenem Verständnis nimmt die Ehre in zweierlei Hinsicht eine Mittelstellung zwischen Moral und Recht ein, nämlich hinsichtlich der Geltungsreichweite und der Verknüpfung von Individuum und sozialer Gruppe. Der Geltungsbereich liegt sozialräumlich in der Mitte zwischen einer als individuellem Gewissen operierenden Moral und dem gesellschaftsweit geltenden Recht. Die Ehre ist dagegen eine Verhaltensvorgabe an die Mitglieder einer Gruppe mittlerer Größe:

Untersucht man [...] die Vorschriften der Ehre auf ihre Inhalte hin, so zeigen sie sich durchgehends als Mittel für die Erhaltung eines sozialen Kreises in seinem Zusammenhalt, seinem Ansehen, der Regelmäßigkeit und Fördersamkeit seiner Lebensprozesse. [...] [D]ie Handlungen und Unterlassungen [...] die die Ehre fordert, offenbaren sich als Zweckmäßigkeiten der Sondergruppierungen, die zwischen dem großen Kreise und dem Individuum stehen. Jede Ehre ist ursprünglich Standesehre, d.h. eine zweckmäßige Lebensform kleinerer Kreise, welche in einem größeren befaßt sind, und durch die Forderung an ihre Mitglieder, die ihr Ehrbegriff deckt, ihre innere Kohäsion, ihren eigentlichen Charakter und ihren Abschluß gegen die anderen Kreise eben desselben größeren Verbandes wahren (Simmel, 600).

Wichtiger als die Bestimmung der Gruppengröße, die irgendwo „zwischen dem großen Kreise und dem Individuum" (ebd.) liegt, d.h. im Bereich von Familie einerseits und Offizierskorps oder Adelsstand andererseits, ist die Form der Bindung. Die Mitglieder bilden einen sozialen ,Körper“ und ihr Zusammenschluss ist insofern als Korporation anzusprechen, als in der Ehr-Verletzung des Einzelnen nicht nur die Ehre der ganzen Gruppe, sondern die Ehre jedes einzelnen Gruppenmitglieds mitangegriffen wird.

Das Ergebnis ist eine besonders ausgeprägte Form der Verkörperung einer sozialen Entität durch den Einzelnen, die zweierlei Besonderheit nach sich zieht. Zum einen erfährt die Verschweißung von Individuum und Gruppe in der korporativistischen Ehre ihre wohl stärkste Zuspitzung. Ungleich stärker noch als im Fall von Kollektivgrößen wie „Nation“ oder „Volk“, die in den Kriegen des 19. und 20. Jahrhunderts ihren Mitgliedern starke Opfer aufbürden, hängt das Leben des Einzelnen an der Erfüllung seiner sozialen Pflicht. Mit den Worten von Georg Simmel ist es die „spezifische Leistung [...] der Ehre“,

6 SIMMEL, Georg: Soziologie. Frankfurt a. M. 2013, S. 602. Von nun an als Simmel zitiert. 
daß sie dem Menschen seine soziale Pflicht zu seinem individuellen Heile macht. Darum gehen der Ehre gegenüber die Aspekte von Recht und Pflicht ineinander über: das Bewahren der Ehre ist so sehr Pflicht, daß man das Recht zu den ungeheuersten Opfern - nicht nur selbstgebrachten, sondern Anderen (sic!) auferlegten, über Andre hinweggehenden - aus ihr zieht (Simmel, 602).

Zum anderen ist es für diese spezifische Form der Regulierung von Interaktionen konstitutiv, dass die Strenge der Anforderungen an den Einzelnen, für die es unter keinerlei Umständen eine Ermäßigung gibt, dadurch vergolten wird, dass die „Ehre“ der Gruppenzugehörigkeit leistungsfrei vergeben wird. Die Ehre in diesem Sinne erwirbt man nicht durch Leistungen, sie ist „garnicht zu erwerben“ (Simmel, 603), sondern darf nur nicht verloren werden. Die im Modus der Ehre konstituierte Gruppe kann „[...] so scheinbar liberal verfahren, weil das ganze, zum Nicht-Verlieren dieses persönlichsten Besitzes [der Ehre; M.Sch.] erforderliche Verhalten gar keinen anderen Inhalt als den Sozialen hat" (ebd.).

Betrachtet man nun wieder die Offiziere Leutnant Gustl und Leutnant Kasda, wird sofort klar, was mit leistungsfreier Vergabe der Ehre einerseits und rein sozialem Ehrgehalt gemeint ist. Überspitzt gesagt, muss man nicht viel können, um an Ehre zu kommen. Alles hängt von den - historisch spezifischen und d.h. um 1900 gelockerten - Rekrutierungsregeln für Offiziere ab. Aber wenn man die Offiziersehre hat, vom Leutnant aufwärts, muss man sie um den Preis seines Lebens verteidigen. Weil sie inhaltlich leer ist, kann sie voraussetzungslos inkludieren. Weil sie aber nur eine Minderheit einschließt, während die Mehrheit draußen bleiben muss, und die wenigen Glücklichen auch noch eine ganze Menge erhalten, ohne dafür vorher etwas geleistet haben zu müssen, kann sie viel fordern, wenn die Exklusivität bedroht ist.

Die Ehre gibt diesen nicht-adligen Leutnants tatsächlich viel. Jedoch ist die Verteidigung eines Status oder von Gütern nicht der Grund dafür, der Ehre sein Leben zu opfern. In einer solchen, d.h. einer implizit individualistischen Perspektive wäre eine Abwägung möglich zwischen dem, was die Ehre gibt und was sie verlangt. Die Kultur der Ehre aber kennt kein Individuum als letzte Entscheidungsinstanz. Die Pointe im zuletzt zitierten Satz von Simmel war, dass die Ehre keinen anderen als sozialen Inhalt hat.

\footnotetext{
Was über den Sinn der Ehre als einer soziologischen Zweckmäßigkeit leicht täuscht, ist gerade der Umstand, mit dem diese Zweckmäßigkeit ihren höchsten Triumph feiert: daß es ihr nämlich gelungen ist, dem Individuum die Bewahrung seiner Ehre als sein innerlichstes, tiefstes, allerpersönlichstes Eigeninteresse zu infundieren. Es gibt vielleicht keinen Punkt, an dem sich das Sozial- und das Individualinteresse derartig verschlingt, wo ein Inhalt, der allein aus dem ersteren verständlich ist, eine imperativische Form angenommen hat, die allein aus dem letzteren zu quellen scheint (Simmel, $601 \mathrm{f}$.).
}

Die Ehre instrumentalisiert das einzelne Mitglied der in ihrem Namen bestehenden Korporation derart vollständig, dass dieses glaubt, sein innerstes Eigeninteresse zu verfolgen, während es nichts anderes tut als eine soziale Institution zu reproduzieren, die 
ihm nichts gibt, außer den äußeren Schein der Ehre. - Wenn das so ist, dann ist, übertragen auf die Literatur, niemand weniger befugt, über die tatsächlichen Ursachen des Handelns aufzuklären als die Figuren. So wenig wie die realen Ehrenmänner, so wenig wissen die Figuren etwas von den sozialen Gründen ihrer vermeintlich individuellen Absichten. Selbst wenn sie zu wissen glauben, dass ihr Ehrenkult „ein Götzendienst“ ist, dem man Folge zu leisten hat, „solange der Götze gilt“, wie die Figur des Geert Innstetten aus Fontanes Roman Effi Briest sagt (die Stelle findet sich am Ende des 27. Kapitels), täuscht sie ihre eigene Begründung.

\section{Textpragmatik und historischer Kontext}

Kommen wir von diesem eigenartigen Ehr-Kodex, „wovon das krasseste Beispiel“ (Simmel, 601) vielleicht gar nicht das Duell ist, sondern der Ehren-Suizid, zurück zu unserem Text. Wenn die Figuren nicht wissen, was sie tun (und die Textintention demnach auch nicht artikulieren oder wenigstens andeuten können), ist es umso wichtiger, den Text als Text, als einheitliches Gefüge aus Erzählertext und Figurenstimmen richtig zu verstehen. Das Erkenntnispotential des exemplifizierenden Geschehens kann nur im Zusammenspiel von Textganzem und Leser aktiviert werden. Die Figuren sind davon entlastet. Dem vorzugsweise dramatischen Modus bei der ,Wiedergabe“ von Gedanken und Reden zum Trotz, sucht man vergebens nach einer Figurenrede, in der die Wahrheit einfach ausgesprochen würde. Nicht einmal in Schnitzlers Drama Freiwild, das der Explikation eines Wissens um die fatale Logik der Ehre am nächsten kommt, gelingt es den Figuren, dieser Logik der Ehre gegenüber in ein objektivierendes Verhältnis zu treten. Modellhafte Objektivierung kann nur der Text in seiner Darstellungsfunktion leisten.

Mittel dazu sind z.B. die narrative Gestaltung wie der autonome Innere Monolog im Falle von Lieutenant Gustl oder die Figurenkonstellation, also ein in der Dimension der dargestellten Welt liegendes Mittel. Letzteres ist hier vorrangig gegeben. Nun zeigt sich, warum es die bereits deutlich gewordene Alternativbildung braucht. In der Relation zwischen Haupthandlung einerseits und den Nebenhandlungen, die das Personal erweitern, steckt das Potential für eine mit literarischen Mitteln in Analogie zu Simmel durchgeführte Analyse. Indem die Nebenfiguren zeigen, dass die enge Kultur der Ehre eine Umwelt hat, in der sich auch leben und zwar gut leben lässt, wenn man nicht satisfaktionsfähig ist, gibt der Text dem Leser zu verstehen, was die Figur prinzipiell nicht wahrnehmen darf, wenn es ihre Funktion ist, das Gefangensein in der Kultur der Ehre zu veranschaulichen.

Historisch gewendet: Die Alternativstruktur, die dem Leser die Aufgabe überlässt, die Möglichkeiten zu sehen, von denen die Figur nichts wissen kann, zeigt den Schwellencharakter an, der dargestellte Welt und historische Wirklichkeit miteinander verbindet. Schnitzlers Ehr-Dramen und -Erzählungen ähnlich wie Fontanes Ehr-Romane, bringen eine Epoche des Übergangs zur Darstellung, indem sie die Hauptfiguren nach Regeln handeln lassen, die im Begriff sind historisch zu werden, wie die Nebenfiguren vorführen. Das Problem ist nur, dass die Texte die neuen Regeln nicht aussprechen können. 
Sie können nicht einfach sagen, was der Fall ist, weil die Regeln einerseits noch in der Genese begriffen sind und es andererseits den Gesetzen der Gattung widerspricht, sie einfach zu proklamieren. Solcher Proklamation steht nicht zuletzt die Tatsache im Weg, dass auch die neue Ordnung, die die alte abzulösen im Begriff ist, ihrerseits nicht ohne Zwänge auskommt.

\section{Figurenkonstellation und Alternativstruktur - Resümee}

Niemand behauptet, dass Schnitzler und noch weniger Fontane utopische Autoren sind, deren Texte von einem implizierten Ideal zwangloser Geselligkeit beseelt sind. Interpretationen erwecken jedoch gelegentlich den Eindruck, als wäre der Zielpunkt jeder Darstellung kulturspezifischer Zwänge wie sie z.B. der Ehr-Kodex an den Tag gelegt, die Aufhebung allen Zwangs. Im Gegensatz dazu möchte ich dafür plädieren, den Autoren ihren politischen und historiographischen Realismus zurückzuerstatten. Schnitzler wie Fontane mit ihrer unbestrittenen Sensibilität für Zwänge, wie sie Geschlechterordnungen oder Ehr-Kodex erzeugen, kritisieren diese nicht von einem imaginären Utopia aus, sondern der nächsten, ihrerseits keineswegs zwanglosen Gesellschaftsordnung her.

In unserem konkreten Fall ist das gut zu sehen. Außer Otto von Bogner, der Parallelfigur zum aktiven Leutnant Kasda gibt es zwei weitere wichtige Nebenfiguren, die über das Motiv des Geldes mit dem primären suizidalen Handlungsstrang verknüpft sind, während sie in der Standeslogik der Ehr-Kultur Außenstehende sind. Beide, der Konsul eines unbekannten Landes und Leopoldine Lebus-Wilram, sind nicht satisfaktionsfähig, verkörpern wie Bogner aber gleichwohl alternative Lebensmöglichkeiten und greifen - ihrem Mangel an Satisfaktionsfähigkeit zum Trotz - tief in Willis Leben ein. Beide haben zudem Geld und stehen dadurch in Opposition zu Willi und Otto. Miteinander stehen sie nicht in Kontakt, aber sie verbindet eine untergründige semantische Relation, denn beide spielen mit dem Spieler. Die Intentionen sind durchaus unterschiedlich. Der Konsul spielt mit Willis Leben, indem er diesem die kürzest mögliche Frist setzt. Aber man muss ihm zugestehen, dass es Willis Kontrollverlust war, der den sinistren Zug am Konsul manifest werden lässt. Bereits am Spieltisch wirkt er bedrohlich. Dass Willi jemandem überhaupt die Chance gibt, ihn regelkonform in die Enge zu treiben, ist primär Willi selbst anzulasten und den Regeln der Kultur, die er verkörpern will, nicht dem Konsul, der damit sein dunkles Spiel treibt.

Leopoldine meint es besser mit Willi - aber nicht ausschließlich besser. Über die Hilfe, die sie ihm zukommen lassen will, lässt sie nichts verlauten, stattdessen nimmt sie sichtbar Rache. Auch dabei geht es interessanterweise um Geld. Geld verwandelt Liebe in Prostitution und würdigt den Akt der Hingabe zu einem Tauschhandel herab. In Form einer Analepse erfährt man, dass unser Leutnant vor einiger Zeit die Gefühle eines Mädchens verletzt hat, das sich ihm aus Liebe hingegeben hat, während er es gedankenlos wie eine Hure benutzt und entlohnt hat. Der Leser erfährt diese genaueren Umstände am Ende, nachdem dieselbe Frau, inzwischen reich, noch einmal mit dem Leutnant Sex hatte. Nun ist es an ihr, seine Situationseinschätzung zu enttäuschen. 
Während er denkt, sich prostituiert zu haben, um die Spielschulden bezahlen zu können, stellt sie eine andere Relation her. Sie lässt ihn im Unklaren darüber, ob er zum vereinbarten Termin leihweise den Geldbetrag von ihr erhalten wird, den er benötigt, um seine Spielschulden zu bezahlen. Stattdessen legt sie einen Tausendguldenschein auf den Tisch, um ihm die Verletzung heimzuzahlen, die er ihr seinerzeit zugefügt hat, als er sie bezahlt hat, während sie Liebe wollte.

Die Kultur des Geldes, das allen Gütern gegenüber, die man mit ihm kaufen kann, gleichgültig ist, trägt den Sieg über die Kultur der Ehre, die keine Äquivalente zulässt, davon. Ihr symbolisch letzter Vertreter besiegelt das eigene Schicksal, ohne darauf zu warten, dass seine Ehrvorstellungen in der übermächtigen Geldkultur endgültig aufgelöst werden. Blickt man auf seine Sexualkontakte, ist der Prozess offensichtlich in vollem Gange. Doch der Leutnant begreift nur die Hälfte. Er erkennt die Rache, vergisst darüber aber, dass längst nicht entschieden ist, ob Leopoldine das Geld leihweise zur Verfügung stellen wird. Er ist auf die Demütigung fixiert. Und weil er die Regeln der Gesellschaft außerhalb der residualen Ehrkultur ignoriert, zieht er daraus die falschen Schlüsse. Er denkt, Leopoldine zöge mit ihrem Racheakt einen Schlussstrich. Sie jedoch stellt damit Symmetrie zwischen ihnen her und schafft damit erst die Voraussetzung dafür, neuerlich irgendeine Form von Beziehung eingehen zu können.

Es kommt wie es kommen muss. Er bringt sich um, bevor das Geld eintrifft. Aber er verwendet die 1000 Gulden, mit denen sie ihn gedemütigt hat, in einer Form die ihm - nun im umgangssprachlichen Sinne - zur Ehre gereicht. Er schickt sein letztes Geld per Kurier zu dem alten Regimentskameraden, der damit rechtzeitig die Veruntreuung kaschieren kann, die am Anfang der Geschichte steht und die Handlung auslöst. Während der Leutnant den Ruf seines ehemaligen Regimentskameraden rettet, der seinen Dienst unehrenhaft quittieren musste, geht er selbst unentehrt in den Tod, obwohl das rettende Geld, dessen Voraussetzung die wechselseitige Herabwürdigung auf Tauschbeziehungen ist, noch rechtzeitig eingetroffen wäre. Schnitzler gestaltet offensichtlich eine gesellschaftliche Transformation, die ein ehemals dominierendes Prinzip sozialer Ordnung durch einen anderes ersetzt. In diesem Zuge werden überkommene Zwänge geschleift, während sich neue Pathologien einspielen.

Das gesellschaftliche Szenario, das diese bescheidene Erzählung imaginiert, ist reizvoll. Bevor man klagt, dass der Leutnant Kasda in der fiktiven Situation, die Schnitzler für ihn ausgeklügelt hat, an einem ganzen Konglomerat an Zwängen zugrunde geht, sollte man fragen, welchen Reim der Text sich auf die miteinander verflochtenen Handlungen macht. Denn während die Ehrlogik mit gradliniger Konsequenz in den Tod führt, ist das Prozedere in der Umwelt, in der sich Gefühle, Sexualität, Freundschaft, Geld und andere Medien des Sozialen überlagern, komplexer und ambivalenter. Und das ist durchaus ein Fortschritt.

Schnitzler kritisiert die Logik der Ehre, die seine reale Lebenswelt immer wieder tangiert hat, mit seinen literarischen Mitteln in aller Schärfe. Er hat erkannt, dass sie in der modernen Gesellschaft, deren zweite historische Phase er miterlebt, einen mit einigen Toten teuer bezahlten Anachronismus darstellt. Aber er ist weit entfernt, die Moderne, die die Ehrlogik bald außer Kraft setzen wird, zur Idylle zu verklären. Auch die moder- 
ne Gesellschaft erzeugt enorme Verhaltenszwänge, diese sind jedoch nicht in gleicher Weise letal. Die modernisierte Moderne pflegt eine andere Beziehung zum Tod. Um in Konfliktsituationen zu einem Ausgleich zu kommen, muss man sich nicht in Lebensgefahr begeben oder anderen Leben ein Ende setzen. Man greift erstmal zum Geld. Das ist weniger schön, denn es nivelliert starke Alternativen, aber es tut auch weniger weh.

PD Dr. Matthias Schöning / matthias.schoening@uni-konstanz.de

Universität Konstanz, Fachbereich Literaturwissenschaft,

78457 Konstanz, DE 
